\title{
BMJ Open Association of postoperative covert stroke and cognitive dysfunction among elderly patients undergoing non-cardiac surgery: protocol for a prospective cohort study (PRECISION study)
}

Qianyu Cui (D) , Dexiang Wang, Min Zeng, Jia Dong, Hailong Jin, Zhengfang Hu, Yuan Zhang, Yuming Peng (D) , Ruquan Han

To cite: Cui Q, Wang $D$, Zeng $\mathrm{M}$, et al. Association of postoperative covert stroke and cognitive dysfunction among elderly patients undergoing non-cardiac surgery: protocol for a prospective cohort study (PRECISION study). BMJ Open 2020;10:e034657. doi:10.1136/ bmjopen-2019-034657

- Prepublication history for this paper is available online. To view these files, please visit the journal online (http://dx.doi. org/10.1136/bmjopen-2019034657).

QC and DW are joint first authors.

Received 30 September 2019 Revised 09 December 2019 Accepted 16 December 2019

D) Check for updates

(C) Author(s) (or their employer(s)) 2020. Re-use permitted under CC BY-NC. No commercial re-use. See rights and permissions. Published by BMJ.

Anesthesiology, Beijing Tiantan Hospital, Capital Medical University, Beijing, China

Correspondence to Dr Yuming Peng;

florapym766@163.com

\section{ABSTRACT}

Introduction The incidence of covert stroke and cognitive dysfunction has gradually increased due to an ageing population. Recently, a prospective cohort study reported perioperative covert stroke was associated with an increased risk of postoperative cognitive dysfunction (POCD) 1 year after non-cardiac surgery. However, the mechanism remains unclear.

Methods and analysis This is a prospective observational trial aiming to investigate the cumulative incidence of perioperative covert stroke and test the hypothesis that perioperative covert stroke associates with POCD in elderly patients undergoing non-cardiac and non-neurological surgery. Data on risk factors, brain MRI, cognitive function evaluation and serum immuneinflammatory cytokines will be collected and analysed. Ethics and dissemination Ethical approval has been granted by the Medical Ethics Committee of Beijing Tiantan Hospital, Capital Medical University (reference number: KY2017-027-02). The results of this study will be disseminated through presentations at scientific conferences and publication in scientific journals. Trial registration number NCT03081429.

\section{INTRODUCTION}

China has entered the period of an ageing society, with the population of over 60 years old estimated to reach $17.2 \%$ by $2020 .{ }^{1}$ Postoperative cognitive dysfunction (POCD) is a common perioperative complication and an independent risk factor for poor prognosis in elderly patients undergoing surgeries. POCD is a syndrome defined as a decrease in preoperative cognitive performance, evaluated by a set of neuropsychological tests, ${ }^{2}$ and was presented by $41.4 \%$ of patients over 60 years old after a major non-cardiac surgery. ${ }^{3} \mathrm{~A}$ prospective observational study by McDonagh et al, ${ }^{4}$ which enrolled 394 elderly patients aged over 55 years old undergoing non-cardiac surgery, found the incidence of
Strengths and limitations of this study

PRECISION is a prospective observational trial aiming to test the hypothesis that perioperative covert stroke associates with postoperative cognitive dysfunction.

- The study will provide new evidence for preventing postoperative cognitive dysfunction if the association between perioperative covert stroke and postoperative cognitive dysfunction could be established.

- The study may not be generalised to other populations since it is a single-centre trial.

POCD at $54.3 \%$ after 6 weeks. Steinmetz et a $\tilde{p}$ also indicated that older age was related to the higher incidence of POCD. In addition, preexisting cognitive impairment, hypotension, neuroinflammatory and endothelial dysfunctions were also associated with POCD. ${ }^{6}$ POCD definitely led to poor quality of life and 5-year cognitive dysfunction after surgery. ${ }^{7}$ However, the mechanism of POCD has not been very clear so far.

Covert stroke is defined as a new infarction of the central nervous system on MRI, without history or symptom of neurological dysfunction attributable to the lesion. ${ }^{8}$ The MRI manifests acute diffusion-weighted imaging (DWI) abnormality and focal T1/ fluid-attenuated inversion recovery (FLAIR) hypointense and T2 hyperintense lesions, with size no less than $3 \mathrm{~mm}$. The prevalence of covert stroke was reported to range from $5 \%$ to $62 \% .^{9}$ The incidence of covert stroke increased from $8 \%$ in individuals aged $60-70$ years old to $22 \%$ in those aged $80-90$ years old. ${ }^{10}$ A strong connection between epidemiological estimates of covert stroke and age was also found. In addition, peripheral immune cells and perioperative hypotension played 
important roles in endothelium injury, ${ }^{11}$ which is also considered a risk factor for stroke. Covert stroke results in recurrent stroke, mortality and cognitive impairment. ${ }^{12}$

Perioperative stroke is a cerebrovascular accident arising from the intraoperative period to 30 days after surgery, and includes overt stroke, transient ischaemic attack and covert stroke. ${ }^{13}$ A systematic review enrolling 16 cohort studies on non-cardiovascular and noncerebrovascular surgeries reported the incidence of perioperative stroke at $0.05 \%-4.4 \%$. However, covert stroke was not included in the review. The incidence of overt stroke was $0.4 \%$, whereas covert stroke was as high as $7 \%$ after non-cardiovascular surgery. ${ }^{1415}$

The Framingham study ${ }^{16}$ showed cerebrovascular diseases, neuroinflammatory and endothelial dysfunctions, abnormal systolic blood pressure, increased body mass index, type II diabetes, stroke history, cardiovascular disease, and atrial fibrillation are also risk factors associated with cognitive impairment, indicating that POCD might be a manifestation of cognitive impairment with covert stroke in elderly patients who received general anaesthesia. A cohort study by Vermeer $\mathrm{t}_{\mathrm{al}} \mathrm{C}^{17}$ assessed the association between covert stroke and cognitive impairment in community populations and indicated that the risk of cognitive impairment in patients with covert stroke was as twice as patients without it. The high-density injury in the periventricular white matter and subcortical atrophy were independently correlated with a cognitive function decline. A prospective cohort study (NeuroVISION) published recently, ${ }^{15}$ which enrolled 1114 elderly patients aged over 65 years old undergoing inpatient, elective, non-cardiac surgery in 12 academic centres in nine countries, showed the incidence of covert stroke was $7 \%$; cognitive decline 1 year after surgery occurred in 29 of $69(42 \%)$ participants who had perioperative covert stroke and in 274 of 932 (29\%) participants who did not have perioperative covert stroke. Patients undergoing general anaesthesia or spinal anaesthesia as well as nerve block were enrolled in the NeuroVISION study. In the PRECISION study, only patients undergoing general anaesthesia will be included. So far, there has been no evidence from Chinese prospective cohort studies examining the association between POCD and covert stroke, especially in the elderly patients after general anaesthesia.

Immune-inflammation response is one of the most important pathogeneses both in stroke and cognitive impairment. ${ }^{18}$ Subcortical cognitive impairment was associated with elevated plasmatic concentration of highsensitivity $\mathrm{C}$ reactive protein, and multiple inflammatory mediators were involved in cognitive impairment after stroke. ${ }^{18}$ Neurofilament light chain (NfL) is a neuronal scaffolding protein which is released into the extracellular space on neuroaxonal damage. Tiedt et al ${ }^{19}$ demonstrated that serum NfL is not only related to cerebral ischaemia, but can also be used as a biomarker to predict the prognosis of neurological function in patients with cerebral ischaemia. NfL is positively related to axonal injury ${ }^{20}$ and has been proven as a biomarker to predict cognitive dysfunction. ${ }^{21}$ More importantly, a recent study indicated that $\mathrm{NfL}$ increased over 48 hours after surgery, which suggested general anaesthesia and surgery might be associated with neuronal damage in the short term. ${ }^{22}$ However, so far few studies have examined NfL in cognitive impairment and covert stroke in elderly patients undergoing non-cardiac, non-neurosurgical surgery after general anaesthesia.

Therefore, our study aims to establish a non-cardiac, non-neurosurgical perioperative covert stroke cohort, observe the cumulative incidence of perioperative covert stroke and associated risk factors, and explore the association between perioperative covert stroke and POCD. Based on previous literature, we propose a hypothesis that perioperative covert stroke is associated with POCD.

\section{METHODS}

\section{Study design}

This is a prospective cohort study. Data will be collected consecutively from patients admitted in non-cardiac, nonneurological wards after written informed consent was obtained.

\section{Objectives}

This trial aims to investigate the cumulative incidence of perioperative covert stroke and observe the association between POCD and perioperative covert stroke in elderly patients undergoing non-cardiac, non-neurosurgical surgery.

\section{Inclusion criteria}

Patients over 60 years old scheduled to undergo elective non-cardiac, non-neurological surgery under general anaesthesia from 2018 to 2020 will be recruited consecutively for eligibility screening.

\section{Exclusion criteria}

The exclusion criteria include history of neurosurgery, depression, epilepsy, traumatic brain injury and MRI contraindications. Patients who are unable to complete the cognitive evaluation and refuse to sign the informed consent will be excluded from the study.

\section{Patient and public involvement}

Patients and the public will not be involved in the development of the research question or the design of the study. Study results will be disseminated by publication in a medical journal and poster presentation at a medical conference.

\section{Anaesthesia management}

Standard routine monitoring will be applied, including non-invasive blood pressure, electrocardiography, pulse oxygen saturation, end-tidal carbon dioxide partial pressure, bispectral index (BIS) and body temperature. Patients will be premedicated with midazolam $0.01-0.03 \mathrm{mg} / \mathrm{kg}$ intravenously $15 \mathrm{~min}$ before induction. Anaesthesia will be induced with sufentanil $(0.1-0.2 \mu \mathrm{g} /$ 
$\mathrm{kg})$, rocuronium $(0.6 \mathrm{mg} / \mathrm{kg})$ or cisatracurium $(0.2 \mathrm{mg} /$ $\mathrm{kg})$, and propofol $(1-2.5 \mathrm{mg} / \mathrm{kg})$ or etomidate $(0.3-$ $0.4 \mathrm{mg} / \mathrm{kg}$ ). Mechanical ventilation will be performed to maintain normocapnia after tracheal intubation. Total intravenous anaesthesia (propofol $4-8 \mathrm{mg} / \mathrm{kg} /$ hour and remifentanil $0.1-0.3 \mu \mathrm{g} / \mathrm{kg} / \mathrm{min})$ or total inhalation anaesthesia (sevoflurane 1.0-1.3 MAC (minimum alveolar concentration)) or intravenous-inhalation combined anaesthesia (propofol 2-3 mg/kg/hour, remifentanil $0.05-0.2 \mu \mathrm{g} / \mathrm{kg} / \mathrm{min}$ and sevoflurane $0.6-1.0 \mathrm{MAC}$ ) will be performed. The type of general anaesthesia will be chosen according to the preference of the anaesthesiologists. The BIS value will be targeted at 40-60 during the surgery. Sufentanil will be administered intermittently to attenuate potent stress responses induced by surgery. Ketamine, lidocaine and dexmedetomidine will not be used routinely. The dosage of anaesthetic drugs and the vital signs will be recorded at $15 \mathrm{~min}$ intervals. Ondansetron will be administered at the end of the surgery to prevent postoperative nausea and vomiting. Neostigmine $(0.04 \mathrm{mg} / \mathrm{kg})$ and atropine $(0.02 \mathrm{mg} / \mathrm{kg})$ will be used to antagonise remnant muscle relaxation if necessary. All patients will be delivered to the postanaesthesia care unit after the surgery. The epidural analgesia or nerve block combined with general anaesthesia will not be applied in the PRECISION study, and the analgesic regimen dosage will be dependent on the anaesthesiologists. The dosage of all perioperative drugs, including specific analgesic regimen of each patient, will be recorded and reported.

\section{Primary outcome}

The primary outcome is the cumulative incidence of perioperative covert stroke, diagnosed by MRI. The standardised brain MRI will be performed at postoperative $5 \pm 2$ days. The MRI sequences include axial FLAIR, gradient echo, T2 and DWI. The DWI sequences enable detection of acute covert stroke that occurs within 10 days of the study. ${ }^{23}$ Therefore, it is unnecessary to obtain preoperative brain MRI to detect new covert brain infarction. The site, amount and size of the ischaemia lesions will be evaluated and recorded with MRI by two trained qualified radiologists who are blinded to the trial. The measurement will be tested by kappa coefficient.

\section{Secondary outcomes}

- Postoperative cognitive function: cognitive assessment will be conducted by trained research members who are blinded to the clinical diagnosis, treatment and MRI. The Mini-Mental State Examination (MMSE) and the Montreal Cognitive Assessment-Basic (MoCA-B) will be used to assess cognitive function 1 day before and 3 months and 1 year after surgery. The SD of each test will be calculated from all of the preoperative scores. Postoperative cognitive dysfunction is defined as at least $2 \mathrm{SD}$ reduction in either the assessment of MMSE or MoCA-B. ${ }^{24}$

- Postoperative delirium: the Confusion Assessment Method for the Intensive Care Unit scale ${ }^{25}$ will be applied to assess delirium 4 hours, 24 hours and 7 days after surgery.

- Recovery quality: the Modified Rankin Scale ${ }^{26}$ and the Lawton Instrumental Activities of Daily Living Scale $^{27}$ will be applied to assess quality of recovery 1 day before surgery, and postoperatively at 30 days and 3 months.

- Biomarkers: peripheral artery blood samples $(4 \mathrm{~mL})$ will be taken at anaesthesia induction and at the end of the surgery, respectively. The blood samples will be stored in vacutainer tubes containing the anticoagulant EDTA and centrifuged at $4^{\circ} \mathrm{C}$. Then the blood samples will be centrifuged for $8 \mathrm{~min}$ at 3000 revolutions per minute, and then pipetted into Eppendorf tubes (Becton Dickinson). The plasma samples will be stored at $-80^{\circ} \mathrm{C}$. All plasma samples will be analysed in the laboratory of Beijing Tiantan Hospital, Capital Medical University by board-certified laboratory technicians blinded to the clinical data. A multiplex panel consisting of hypersensitive $\mathrm{C}$ reactive protein and $\beta$ amyloid will be applied. NfL will be tested by fourthgeneration (single-molecule array) assays. Fourthgeneration assays permit highly sensitive, longitudinal detection of blood NfL level both in patients with cognitive decline and with stroke. ${ }^{28} 29$

- Quality of life: the quality of life will be assessed using the EuroQol-5D scale 1 day before surgery, and 30 days and 3 months after surgery.

- Depression state: the state of depression will be assessed using the Geriatric Depression Scale ${ }^{30} 1$ day before surgery, and at 30 days and 3 months after surgery.

- The incidence of comorbidity including myocardial infarction, cardiac arrest, pulmonary embolism, sepsis, surgical site infection and persistent postoperative pain will be recorded within the first 30 days and 3 months after surgery.

\section{Sample size estimates}

Based on previous literature, the incidence of POCD at 1 week after surgery and perioperative covert stroke is approximately $20 \%{ }^{31}$ and $10 \%,{ }^{23}$ respectively, in patients over 60 years old undergoing non-cardiac surgery. In the current study, the accuracy of cumulative incidence in POCD and covert stroke is set at $5 \%$ and $10 \%$, and the sample size is estimated to be 1022 and 593, respectively, with $\alpha$ at 0.05 .

A multivariate analysis will be used to investigate the association between serum immune-inflammatory cytokines and postoperative 3-month cognitive impairment or covert stroke. It is estimated that 10 independent variables should be included in the analysis, so 100 patients with cognitive impairment will be needed. ${ }^{32}$ Considering the incidence of POCD is approximately $20 \%$ and covert stroke is about $10 \%$ in elderly patients, the total sample size will be 500 and 1000 , respectively. The total sample size will be set at 1100 to meet all the above requirements. 


\section{Statistical analyses}

Categorical data will be expressed as number and percentage and analysed using $\chi^{2}$ test or Fisher's exact test. Continuous data will be expressed as mean and SD or IQR and analysed using Mann-Whitney U test or independent t-test. To determine the relationship between perioperative variables and the development of covert stroke, univariate analysis will be performed using logistic regression. Variables that are statistically significant $(p<0.1)$ in the univariate analysis will then be included as independent variables in the multivariate logistic regression analysis to determine the independent risk factors for postoperative covert stroke. The association between perioperative covert stroke and POCD will be analysed by relative risk, attributable risk per cent, population attributable risk and multiple logistic regression. Subgroup analysis will be performed based on age, intraoperative blood pressure, history of stroke, type of surgery and duration of anaesthesia. Cox proportional hazards model will be applied to determine the incidence of 1-year overt stroke and death between patients who suffered from perioperative covert stroke and patients who have not had perioperative covert stroke. The aetiology (including serum NfL levels) of covert stroke and POCD will be analysed by risk ratio and logistic regression.

\section{Adverse events}

Adverse events include burns, endovascular stent shift and orthopaedic implant shift caused by magnetic resonance examination. All adverse events will be monitored and recorded. The chief investigator will be responsible for all adverse events reported. Any adverse event will be reported immediately to the endpoint adjudication committee, who will investigate the severity and causality of the adverse events. The incidence of adverse events will be summarised for each group and compared using $\chi^{2}$ test or Fisher's exact test.

\section{DISCUSSION}

The study is a prospective observational trial aiming to investigate the cumulative incidence of perioperative covert stroke, which is closely associated with POCD. The incidence and risk factors will be analysed by neuroimaging, cognitive function evaluation and serum immunological factors level.

The safety issues surrounding MRI examination after surgery should be considered. Several studies have shown that MRI can be performed after stent implantation. ${ }^{33}$ It has been reported that it is safe to implement MRI examinations in patients with implanted or temporary cardiovascular devices. ${ }^{34}$ Mrkobrada et $a l^{23}$ performed a prospective cohort study to develop a preliminary estimate of the incidence of postoperative covert stroke. In this trial, brain MRI was performed in patients who underwent non-cardiac surgery 3 and 10 days after surgery with no adverse events. Schubert $e t a l^{35}$ and Bernstein $e t a l^{36}$ also found it was safe and effective to conduct
MRI scan after spinal surgery. Ex vivo testing has been used to evaluate MRI-related heating for various metallic implants and materials. ${ }^{37}$ It has been reported that only minor temperature changes are associated with the MRI procedure. ${ }^{38}$ Although MRI examination is not allowed for some spinal implants, the procedure is safe for most patients with orthopaedic implants. ${ }^{39}$ In the present study, the researchers will check the instructions with regard to the implants and then decide whether an MRI scan can be performed after surgery.

The diagnostic criteria for POCD remain controversial. Neuropsychological testing of cognitive function before and after surgery is the most reliable and common diagnostic method. ${ }^{40}$ MMSE is the most widely used tool as it accurately reflects mental status and cognitive function. It focuses on the cognitive function and eliminates the interference of emotions and consciousness. However, MMSE is not an instrument recommended to identify mild cognitive impairment (MCI).$^{41}$ The Montreal Cognitive Assessment (MoCA) is a cognitive function assessment tool developed in 2004 and has the advantage of screening for MCI. ${ }^{42}$ However, the results of the MoCA scale are significantly affected by the level of education. The patients recruited in the PRECISION study are elderly patients in China with an education level that is generally low. Julayanont $e t$ al in 2014 published a new version of the scale called MoCA-B to specifically evaluate elderly patients with low education level and are illiterate. ${ }^{43}$ Saleh $e t a t^{44}$ and Chen $e t a t^{45}$ performed two large clinical trials which demonstrated that MoCA-B had good validity and internal consistency in discriminating between normal and ill elderly patients. Therefore, in this study, MoCA-B will be chosen instead of MoCA as a tool to evaluate cognitive function.

\section{Summary}

In summary, the study aims to investigate the cumulative incidence of perioperative covert stroke and test the hypothesis that POCD is associated with postoperative covert stroke. If the result of the PRECISION study is positive, it will provide evidence for the prevention and treatment of POCD and covert stroke in non-cardiac and non-neurological surgical patients over 60 years old.

\section{Dissemination}

The results of this study will be disseminated through presentations at scientific conferences and publication in scientific journals.

\section{Informed consent}

Patients who are eligible for the trial will be given informed consent form by a member of the research team. All patients will be given ample time to consider participation in the trial. A completed informed consent form is required for enrolment in the trial. Patients who agree to participate in the PRECISION study and sign the informed consent will be involved in the study. The 
investigators must keep the original signed consent form as well as an additional copy of this form.

\section{Timeline}

The study will take approximately 3 years to complete enrolment and outcome assessment. The recruitment started on 23 May 2018. The anticipated completion date will be 30 September 2020 .

\section{Audits}

The data monitoring committee will conduct audits through regular interviews, letters or telephone. The data monitoring committee reserves the right to audit the recruitment of patients at any time. The auditing process will be independent from the investigators.

\section{Amendments to the protocol}

Amendments to the protocol will only be made by the academic committee and with the approval of the Medical Ethics Committee, Beijing Tiantan Hospital, Capital Medical University. Any modifications will be recorded and applied to all subsequent patients. The registration record will be updated as well.

Contributors QC and DW were involved in the conception and design, data collection and analysis, and manuscript writing. MZ, HJ, DW, JD, ZH and YZ were involved in the conception and design, data collection, and manuscript revision. $\mathrm{RH}$ and YP were involved in the conception and design, data analysis, and manuscript revision. RH and YP contributed equally to the work. All authors have read and approved the final manuscript.

Funding This work was supported by funding from the National Key Research and Development Program of China (Grant No. 2018YFC2001901), Capital Development Research Fund Project (2018-2-2044), Incubating Program (PX2018023) and Ascent Plan (DFL20180502) from the Beijing Municipal Administration of Hospitals.

Competing interests None declared.

Patient consent for publication Not required.

Ethics approval Ethical approval has been granted by the Medical Ethics Committee of Beijing Tiantan Hospital, Capital Medical University (reference number: KY2017-027-02). The patients who agree to participate in the PRECISION study and sign the informed consent will be included. The outcome results will not be discussed or presented outside the trial group unless authorised by the Medical Ethics Committee. Compensation for ancillary and post-trial care will be provided through funding

Provenance and peer review Not commissioned; externally peer reviewed.

Open access This is an open access article distributed in accordance with the Creative Commons Attribution Non Commercial (CC BY-NC 4.0) license, which permits others to distribute, remix, adapt, build upon this work non-commercially, and license their derivative works on different terms, provided the original work is properly cited, appropriate credit is given, any changes made indicated, and the use is non-commercial. See: http://creativecommons.org/licenses/by-nc/4.0/.

\section{ORCID iDs}

Qianyu Cui http://orcid.org/0000-0002-6102-1708

Yuming Peng http://orcid.org/0000-0002-2630-2467

\section{REFERENCES}

1 YPS J. Aging process in China and its influences on social economy. Chin J Geriatr 2014;33:113-5.

2 Berger M, Nadler JW, Browndyke J, et al. Postoperative cognitive dysfunction: minding the gaps in our knowledge of a common postoperative complication in the elderly. Anesthesiol Clin 2015;33:517-50.
3 Monk TG, Weldon BC, Garvan CW, et al. Predictors of cognitive dysfunction after major noncardiac surgery. Anesthesiology 2008;108:18-30.

4 McDonagh DL, Mathew JP, White WD, et al. Cognitive function after major noncardiac surgery, apolipoprotein E4 genotype, and biomarkers of brain injury. Anesthesiology 2010;112:852-9.

5 Steinmetz J, Christensen KB, Lund T, et al. Long-term consequences of postoperative cognitive dysfunction. Anesthesiology 2009;110:548-55.

6 Riedel B, Browne K, Silbert B. Cerebral protection: inflammation, endothelial dysfunction, and postoperative cognitive dysfunction. Curr Opin Anaesthesiol 2014;27:89-97.

7 Steinmetz J, Rasmussen LS. Peri-operative cognitive dysfunction and protection. Anaesthesia 2016;71:58-63.

8 Sacco RL, Kasner SE, Broderick JP, et al. An updated definition of stroke for the 21st century: a statement for healthcare professionals from the American Heart Association/American Stroke Association. Stroke 2013;44:2064-89.

9 Fanning JP, Wong AA, Fraser JF. The epidemiology of silent brain infarction: a systematic review of population-based cohorts. BMC Med 2014;12:119.

10 Vermeer SE, den Heijer T, Koudstaal PJ, et al. Incidence and risk factors of silent brain infarcts in the population-based Rotterdam Scan Study. Stroke 2003;34:392-6.

11 Dirnagl U. Pathobiology of injury after stroke: the neurovascular unit and beyond. Ann N Y Acad Sci 2012;1268:21-5.

12 Gupta A, Giambrone AE, Gialdini G, et al. Silent brain infarction and risk of future stroke: a systematic review and meta-analysis. Stroke 2016;47:719-25.

13 Macellari F, Paciaroni M, Agnelli G, et al. Perioperative stroke risk in nonvascular surgery. Cerebrovasc Dis 2012;34:175-81.

14 Kikura M, Bateman BT, Tanaka KA. Perioperative ischemic stroke in non-cardiovascular surgery patients. J Anesth 2010;24:733-8.

15 NeuroVISION Investigators. Perioperative covert stroke in patients undergoing non-cardiac surgery (NeuroVISION): a prospective cohort study. Lancet 2019;394:1022-9.

16 Satizabal CL, Beiser AS, Chouraki V, et al. Incidence of dementia over three decades in the Framingham heart study. N Engl J Med 2016;374:523-32.

17 Vermeer SE, Prins ND, den Heijer T, et al. Silent brain infarcts and the risk of dementia and cognitive decline. $N$ Engl J Med 2003;348:1215-22.

18 McColl BW, Allan SM, Rothwell NJ. Systemic infection, inflammation and acute ischemic stroke. Neuroscience 2009;158:1049-61.

19 Tiedt S, Duering M, Barro C, et al. Serum neurofilament light: a biomarker of neuroaxonal injury after ischemic stroke. Neurology 2018;91:e1338-47.

20 Magnoni S, Esparza TJ, Conte V, et al. Tau elevations in the brain extracellular space correlate with reduced amyloid- $\beta$ levels and predict adverse clinical outcomes after severe traumatic brain injury. Brain 2012:135:1268-80.

21 Mattsson N, Cullen NC, Andreasson U, et al. Association between longitudinal plasma neurofilament light and neurodegeneration in patients with Alzheimer disease. JAMA Neurol 2019;76:791-9.

22 Evered L, Silbert B, Scott DA, et al. Association of changes in plasma neurofilament light and tau levels with anesthesia and surgery: results from the capacity and ARCADIAN studies. JAMA Neurol 2018;75:542-7.

23 Mrkobrada M, Hill MD, Chan MTV, et al. Covert stroke after non-cardiac surgery: a prospective cohort study. Br J Anaesth 2016;117:191-7.

24 Evered L, Silbert B, Knopman DS, et al. Recommendations for the nomenclature of cognitive change associated with anaesthesia and surgery-2018. Br J Anaesth 2018;121:1005-12.

25 Alcoba Pérez Á, Ciria Poza S, Carracedo Catalán C, et al. [Assessment of concordance between CAM-ICU scale and nursing delirium scale in postoperative period of ICU patients after cardiac surgery]. Enferm Intensiva 2014;25:100-6.

26 Broderick JP, Adeoye O, Elm J. Evolution of the modified Rankin scale and its use in future stroke trials. Stroke 2017;48:2007-12.

27 Jimenez-Caballero PE, Lopez-Espuela F, Portilla-Cuenca JC, et al. Evaluation of the instrumental activities of daily living following a stroke by means of the Lawton and Brody scale]. Rev Neurol 2012:55:337-42.

28 Khalil M, Teunissen CE, Otto M, et al. Neurofilaments as biomarkers in neurological disorders. Nat Rev Neurol 2018;14:577-89.

29 Kuhle J, Barro C, Andreasson U, et al. Comparison of three analytical platforms for quantification of the neurofilament light chain in blood samples: ELISA, electrochemiluminescence immunoassay and Simoa. Clin Chem Lab Med 2016;54:1655-61. 
30 Ishihara T, Terada S. [Geriatric Depression Scale (GDS)]. Nihon Rinsho 2011;69:455-8.

31 Rasmussen LS, Johnson T, Kuipers HM, et al. Does anaesthesia cause postoperative cognitive dysfunction? a randomised study of regional versus general anaesthesia in 438 elderly patients. Acta Anaesthesiol Scand 2003;47:260-6.

32 Wilson Van Voorhis CR, Morgan BL. Understanding power and rules of thumb for determining sample sizes. Tutor Quant Methods Psychol 2007;3:43-50.

33 Levine GN, Gomes AS, Arai AE, et al. Safety of magnetic resonance imaging in patients with cardiovascular devices: an American heart association scientific statement from the Committee on diagnostic and interventional cardiac catheterization, Council on clinical cardiology, and the Council on cardiovascular radiology and intervention: endorsed by the American College of cardiology Foundation, the North American Society for cardiac imaging, and the Society for cardiovascular magnetic resonance. Circulation 2007;116:2878-91.

34 Hundley WG, Bluemke DA, Finn JP, et al. ACCF/ACR/AHA/NASCI/ SCMR 2010 expert consensus document on cardiovascular magnetic resonance: a report of the American College of cardiology Foundation Task force on expert consensus documents. J Am Coll Cardiol 2010:55:2614-62.

35 Schubert GA, Diepers M, Hegewald AA, et al. Routine postoperative imaging early after lumbar decompression surgery: a prospective evaluation. Spine 2013;38:E1263-8.

36 Bernstein P, Hentschel S, Platzek I, et al. The assessment of the postoperative spinal alignment: MRI adds up on accuracy. Eur Spine J 2012;21:733-8.
37 Diaz FL, Tweardy L, Shellock FG. Cervical external immobilization devices: evaluation of magnetic resonance imaging issues at 3.0 Tesla. Spine 2010;35:411-5.

38 Shellock FG, Hatfield M, Simon BJ, et al. Implantable spinal fusion stimulator: assessment of Mr safety and artifacts. J Magn Reson Imaging 2000;12:214-23.

39 Thakkar RS, Malloy JP, Thakkar SC, et al. Imaging the postoperative spine. Radiol Clin North Am 2012;50:731-47.

40 Polunina AG, Golukhova EZ, Guekht AB, et al. Cognitive dysfunction after on-pump operations: neuropsychological characteristics and optimal core battery of tests. Stroke Res Treat 2014;2014:1-18.

41 Beyermann S, Trippe RH, Bähr AA, et al. [Mini-Mental State Examination in geriatrics: An evaluation of diagnostic quality]. $Z$ Gerontol Geriatr 2013:46:740-7.

42 Tan J-ping, Li N, Gao J, et al. Optimal cutoff scores for dementia and mild cognitive impairment of the Montreal cognitive assessment among elderly and oldest-old Chinese population. J Alzheimers Dis 2015;43:1403-12.

43 Julayanont $\mathrm{P}$, Tangwongchai S, Hemrungrojn S, et al. The Montreal cognitive Assessment-Basic: a screening tool for mild cognitive impairment in Illiterate and Low-Educated elderly adults. J Am Geriatr Soc 2015;63:2550-4.

44 Saleh AA, Alkholy RSAEHA, Khalaf OO, et al. Validation of Montreal cognitive Assessment-Basic in a sample of elderly Egyptians with neurocognitive disorders. Aging Ment Health 2019;23:551-7.

45 Chen K-L, Xu Y, Chu A-Q, et al. Validation of the Chinese version of Montreal cognitive assessment basic for screening mild cognitive impairment. J Am Geriatr Soc 2016;64:e285-90. 